\title{
Original Article \\ Antibacterial effect of Medicago sativa extract on the common bacteria in sinusitis infection
}

\author{
Hamid Chegini ${ }^{1}$, Mozhgan Oshaghi ${ }^{*}$, Mohammad Amin Boshagh ${ }^{3}$, Poorya Foroutan ${ }^{3}$, Amir Hossein Jahangiri ${ }^{1}$
}

\section{ARTICLE INFO}

Article History:

Received 27 December 2017

Revised 5 January 2018

Accepted 5 January 2018

Published online 13 January 2018

\section{Keywords:}

Medicinal plants;

Medicago sativa;

Antibacterial effect;

Sinusitis

${ }^{1}$ Department of Immunology, Tarbiat Modares University, Tehran, Iran

${ }^{2}$ Department of Microbiology, Faculty of Paramedical, Iran University of

Medical Sciences, Tehran, Iran

${ }^{3}$ Student Research Committee,

Kurdistan University of Medical

Sciences, Sanandaj, Iran

*Correspondence:

Dr Mozhgan Oshaghi, Faculty of

Paramedical, Iran University of

Medical Sciences, Tehran, Iran

Email: mozghan.oshaghi@yahoo.com

\begin{abstract}
Introduction: Sinusitis is a serious health hazard that is caused by inflammation of the surrounding sinus. Bacterial infection due to Streptococcus pneumonia microorganisms, Haemophilus influenza, Moraxella catarrhalis and Staphylococcus aureus is one of the factors causing sinusitis and bronchitis. Medicago sativa is known as a precious medicinal plant. The purpose of this study is to investigate the antibacterial effect of Medicago sativa extract on common bacteria in sinusitis and bronchitis.

Methods: In this experimental study, after root extraction of the plant by maceration method, first the quantitative Minimum Inhibitory growth Concentration (MIC) test was carried out and then disc diffusion quality test was performed with the observance of standard strains for Streptococcus pneumonia, Haemophilus influenza, Moraxella catarrhalis, and Staphylococcus aureus.

Result: The results showed that MIC of Medicago sativa root extract against Streptococcus pneumoniae, Haemophilus influenza and Moraxella catarrhalis was $125 \mathrm{mg} / \mathrm{ml}$. Furthermore, the extract did not affect Staphylococcus aureus, and in all of the tubes turbidity and growth were observed. In the disc diffusion method, the diameter of the inhibition zone was $16 \mathrm{~mm}$ for Moraxella catarrhalis, $13 \mathrm{~mm}$ for Streptococcus pneumoniae and $10 \mathrm{~mm}$ for Haemophilus influenza, and for Staphylococcus aureus, no inhibition zone was found around the disk containing the extract.

Conclusion: The results of this study showed the inhibitory effect of Medicago sativa root extract on Streptococcus pneumoniae, Haemophilus influenza and Moraxella catarrhalis. By extracting the important compounds of this plant, we can hope to make a suitable drug for the treatment of sinusitis.
\end{abstract}

\section{Introduction}

$\mathrm{S}_{\mathrm{s}}^{\mathrm{i}}$ inusitis is a serious threat to human health, and the social and economic burden imposes heavy costs on the affected person and the community (1).
Sinusitis is the inflammation of sinuses around nose, the causes of which can be infections, allergic reactions, as well as self-contained disorders. Bacterial sinusitis is divided into two acute and chronic groups, each of which runs its own path (2).

This is an Open Access article distributed under the terms of the Creative Commons Attribution License (https://creativecommons.org/licenses/by/4.0/), which permits unrestricted use, distribution, and reproduction in any medium, provided the original work is properly cited. 
Bronchitis is associated with the inflammation of bronchus, with symptoms such as choking, coughing, and phlegm (3). Approximately, 75\% of outpatient visits to physicians who need antibiotics are due to sinusitis and bronchitis. The isolated bacterial agents from most of these patients include Streptococcus pneumoniae, Haemophilus influenza, Moraxella catarrhalis and Staphylococcus aureus (4-6). Sinusitis treatment involves removing predisposing factors (allergic rhinitis, immunodeficiency, diabetes, depression of the nasal septum, nasal congestion, concha bullous, anemia and malnutrition), nasal wash with normal saline, inside nose corticosteroid and oral antibiotics. Cephalosporins, macrolides, and amoxiclavare common used antibiotics (7). The improper use of antimicrobial chemicals leads to increased antibiotic resistance in most bacteria (8). The resistance of bacteria to antibiotics is increasing day to day, which makes humans think of replacing effective antimicrobials with fewer side effects. Herbal extracts contain substances that can be used against many microorganisms. These antimicrobial effects have been demonstrated against bacteria and fungi (9). The Medicago Sativa known as alfalfa. Medicago Sativa is a medicinal plant belonging to Fabaceae family. This herb is rich from substances called saponins. Recent studies have shown that saponins produce many effects in the body that can boost the immune system, have anti-tumor effects, helps detoxification due to the combination with bile acid, as well as antimicrobial effects such as antibacterial, antifungal and also has an antiviral effect $(10,11)$. In most cases, numerous scientific investigations have led to the confirmation of therapeutic value of medicinal plants. Medicago Sativa used to be known/named as the Queen of Plants and Green Gold (12). The plant is rich in various proteins, calcium, mineral elements, various vitamins in group $\mathrm{B}$, vitamins $\mathrm{A}, \mathrm{C}, \mathrm{D}, \mathrm{E}, \mathrm{K}$ and amylase enzyme, emulcer, invertase and pectinase, as well as very small amounts of arsenic and silica (13-17). In the taste of people, there is a greater tendency to use plant compounds instead of chemical drugs. while, many researchers believe that the side effects of taking herbal drugs are much fewer than chemical drugs. Due to the wide distribution of medicinal plants in our country, studies on their antimicrobial properties provide a suitable basis for using the results of these studies to replace the natural drugs to control and treat bacterial infections. It can reduce the use of chemical drugs and their complications. In this research, the antimicrobial effects of alfalfa extract on common bacteria in sinusitis and bronchitis are investigated.

\section{Methods}

\section{Bacterial strains}

In this research, bacterial strains of Streptococcus pneumonia (ATCC49619), Haemophilus influenza (ATCC35056), Moraxella catarrhalis (ATCC25240) and Staphylococcus aureus (ATCC25923) are tested, which were prepared by Pasteur Institute.

\section{Culture medium}

Blood agar culture media, chocolate agar and Mueller hinton broth were used in this study, which were purchased from Merck, Germany.

\section{Extract preparation}

The extract used was an extract from Medicago Sativa roots which was prepared by maceration method.

The roots of Medicago Sativa were obtained from Robat Karim, Tehran, Iran and confirmed by botanical experts. The roots of the plant in were kept a dark and humid environment, and then were milled. After, we weighed $100 \mathrm{~g}$ of the root stock powder and then added 400 CC $80 \%$ methanol to the powder and placed on a shaker at $4000 \mathrm{rpm}$ for 24 hours. Next, the supernatant was removed and again $300 \mathrm{cc}$ methanol was added to the residue. The solution was placed inside a refrigerator and this process was repeated three times. Then, the collected solution was coated with Buchner Funnel by means of a vacuum distillation machine by maceration method using a Hiedolph retro-device (Hiedolphcompany, Germany), at $40{ }^{\circ} \mathrm{C}$ and $210-290$ $\mathrm{rpm}$. To separate water from the extract, a Lyophilized device was used and the extract was placed in the apparatus for 48 hours. After determining the dry weight of the extract, a concentration of $1000 \mathrm{mg} / \mathrm{ml}$ was prepared and the extract was stored in refrigerator for later usage.

\section{Preparation of test microorganisms}

At first, standard strains' samples were placed on blood agar and chocolate agar culture medium and the samples were kept at $37^{\circ} \mathrm{C}$ for 24 hours. Subsequently, a quantitative minimum inhibitory concentration (MIC) test and a disc diffusion test for samples with Medicago Sativa extract was laid on Mueller hinton broth medium.

\section{Determination of Minimum Inhibitory Concentration}

After preparing the microbial suspension using the medium of the Mueller hinton broth according to the McFarland standards, a diluted equivalent (1000 mg / $\mathrm{ml}$ ) of the extract was obtained using sterilized distilled 
water (18). Next $0.5 \mathrm{ml}$ of Mueller hinton broth was added to 10 sterilized tubes, and in the next step, after shaking, $0.5 \mathrm{ml}$ diluted Medicago Sativa extract was added tube No.2, after shaking in tube No.2, $0.5 \mathrm{ml}$ of the solution was transferred to tube No.3 and the process was repeated to tube No.9 and finally, $0.5 \mathrm{ml}$ of the solution was discarded (the concentration of the first tube was $500(\mathrm{mg} / \mathrm{ml})$. After this step, according to the McFarland standards, $0.5 \mathrm{ml}$ of the microbial suspension was added to all the tubes and were kept at $37{ }^{\circ} \mathrm{C}$ for 24 hours. Eventually, the first transparent tube with inhibited bacterial growth was considered MIC or the least concentration of growth inhibitor of the extract. To confirm a tube before the first transparent tube and the next tube was cultured on a chocolate agar medium. Each treatment had a positive (culture medium with bacteria of each strain) and negative (extract-free culture medium and a bacterium) control. In this test, the tube No.1 was negative and the tube No.10 was positive.

\section{Determination disc diffusion}

In order to test the Disc diffusion (DD), the bacteria were grown on Mueller hinton broth. Discs with diameter of $10 \mathrm{~mm}$, impregnated with Medicago sativa root extract (MIC equivalent) were placed on the medium. After 24 hours of incubation at $37^{\circ} \mathrm{C}$, the diameter of the inhibition zone was measured from the back of the plate with the ruler and the results were recorded (19). Moreover, in the case of haemophilus influenza from the medium of Muller Hinton Broth with Growth factors (supplement) containing 3\% of sheep blood lysis was used (20).

\section{Results}

In this study, the effect of Medicago sativa root extract on standard strains of Streptococcus pneumoniae, Haemophilus influenza, Moraxella catarrhalis and Staphylococcus aureus was investigated. The results show that the MIC of Medicago sativa root extract against Streptococcus pneumoniae, Haemophilus influenza and Moraxella catarrhalis was $125 \mathrm{mg} / \mathrm{ml}$, that is, MIC of Medicago sativa roots extract at this concentration has an effect. The extract with the studied concentration and used method did not affect Staphylococcus aureus, and turbidity and growth were observed in all tubes (Table 1). After determining the MIC for the bacteria in the vicinity of the Medicago sativa root extract, a disc diffusion test was laid. In the disc diffusion method, the diameter of the inhibition zone was $16 \mathrm{~mm}$ for Moraxella catarrhalis, $13 \mathrm{~mm}$ for Streptococcus pneumoniae and $10 \mathrm{~mm}$ for
Haemophilus influenza and in the case of Staphylococcus aureus, no inhibition zone was found around the disc containing the extract, which confirms the results of the MIC method data (Table $1)$.

\section{Discussion}

According to the results, the Medicago sativa root extract has a significant antibacterial effect on common bacteria in sinusitis and bronchitis including Streptococcus pneumoniae, Haemophilus influenza and Moraxella catarrhalis, but has no effect on Staphylococcus aureus bacteria. One of the possible reasons for the lack of anti-bacterial effect of Medicago sativa roots on Staphylococcus aureus may be the weakness of the active antibacterial agent Medicago sativa extract on the bacteria. Another factor that may affect the antibacterial effects of an extract of a plant is the extraction method and solvent type (21). Extracts with different methods and solvents from one plant can exhibit different antibacterial effects on a particular bacterium (22). In a study by Chavan et al. performed in India in 2015, they examined the antibacterial effect of Medicago sativa extract. In this study, the antibacterial effects of Medicago sativa extract on E.coli and Pseudomonas aeruginosa were studied by MIC and disk diffusion methods. The results showed inhibitory and antibacterial effect of Medicago sativa extract on the bacterial growth (23). In a study by George p et al., performed in India in 2014, the antibacterial effect of Medicago sativa extract on different bacteria such as Bacillus licheniformis, Pseudomonas aeruginosa, Lactococcus lactis, Klebsiella pneumonia, Bacillus cereus was investigated. In this study, MIC and disc diffusion methods were used to study the inhibitory effect of Medicago sativa plant extract. The results of this study, such as the previous study (Chavan et al.), show the effectiveness of the anti-bacterial effect of Medicago sativa extract on the bacteria mentioned (10). Various studies have shown that Medicago Sativa has antimicrobial activity against bacteria (24, 25). Also, in a similar study carried out by Aliahmadi et al. in 2011, the effect of this extract on the elimination of bacteria was confirmed (26). Certainly, in the ancient medicine, special attention was paid to the various functions of plant extracts based on the organs involved in a disease. Many plants have secondary metabolites, such as terpenes, alkaloids, glycosides, flavonoids, etc., of which secondary metabolites have the ability to fight against microorganisms (27) 
Table 1. The results of MIC and inhibition

\begin{tabular}{|c|c|c|c|c|}
\hline Microorganism & M.catarrhalis & S.pneumonia & H. influenzae & S.aureus \\
\hline \multicolumn{5}{|l|}{ Extract } \\
\hline Medicago sativa MIC (mg /ml) & 125 & 125 & 125 & No effect \\
\hline $\begin{array}{l}\text { Medicago sativa inhibition } \\
\text { zone }(\mathrm{mm})\end{array}$ & 16 & 13 & 10 & No Zone \\
\hline
\end{tabular}

\section{Conclusion}

The results of this study indicate the inhibitory effect of Medicago Sativa root Streptococcus pneumoniae, Haemophilus influenza and Moraxella catarrhalis. Plants can be considered as a source of potential chemical substances, only a part of which has been exploited. In future studies, researchers should separately investigate the effects of components in Medicago sativa roots extract in terms of antimicrobial effects. A clinical evaluation is also needed to find out that it can be used as a cheap substance and with low side effects to prevent and treat common infections, especially in cases where drug resistance and the risk of recurrent infections are high. The results of this study suggest that Medicago sativa root extract can be used alone or in combination with other antimicrobial agents to improve or treat sinusitis and bronchitis. With this and other studies, we can hope to develop a suitable drug for the treatment of sinusitis. However, it is needed to perform this study in invivo conditions to evaluate the probable toxicity of the extract due to the high levels of arsenic and silica (9),its properties and the appropriate concentrations of this extract for living organisms.

\section{Ethical disclosure}

In this study, blood samples or tests that threatened the health of individuals were not used.

\section{Acknowledgements}

Thanks to the assistance of the Iran University of Medical Sciences (Microbiology Labratory)

\section{Author Contributions}

All the authors have accepted responsibility for the entire content of this submitted manuscript and approved submission.

\section{Conflict of interest}

The authors declare that they have no conflict of interest.

\section{Funding/Support \\ None declared.}

\section{References}

1. Boase S, Foreman A, Cleland E, Tan L, Melton-Kreft R, Pant $\mathrm{H}$, et al. The microbiome of chronic rhinosinusitis: culture, molecular diagnostics and biofilm detection. BMC infectious diseases. 2013;13(1):210. doi: 10.1186/14712334-13-210.

2. Rudmik L, Soler ZM. Adult Chronic Sinusitis. Jama. 2015;314(9):964. doi: 10.1001/jama.2015. 7892.

3. Macfarlane J, Holmes W, Gard P, Macfarlane R, Rose $\mathrm{D}$, Weston V, et al. Prospective study of the incidence, aetiology and outcome of adult lower respiratory tract illness in the community. Thorax. 2001;56(2):109-114. doi: 10.1136/thorax.56.2.109

4. Kasper DL, Braunwald E, Fauci A, Hauser S, Longo D, Jameson JL. Harrison's principles of internal medicine. New York. 2005:1467-8.

5. Fendrick AM, Saint S, Brook I, Jacobs MR, Pelton S, Sethi S. Diagnosis and treatment of upper respiratory tract infections in the primary care setting. Clinical therapeutics. 2001;23(10):1683-1706. PMID: 11726004

6. Bhattacharyya N, Kepnes LJ. The microbiology of recurrent rhinosinusitis after endoscopic sinus surgery. Arch Otolaryngol Head Neck Surg. 1999;125(10):111720. PMID: 10522504

7. Larrondo J, Agut M, Calvo-Torras M. Antimicrobial activity of essences from labiates. Microbios. 1995;82(332):171-2. PMID: 7630324

8. Weinstein RA. Controlling antimicrobial resistance in hospitals: infection control and use of antibiotics. Emerg Infect Dis. 2001;7(2):188-192.

9. Lin J, Opoku A, Geheeb-Keller M, Hutchings A, Terblanche S, Jäger AK, et al. Preliminary screening of some traditional zulu medicinal plants for antiinflammatory and anti-microbial activities. J Ethnopharmacol. 1999;68(1):267-74. PMID: 10624887

10. Joy GS, George P. Antimicrobial screening of Alfalfa (Medicago sativa) in various bacterial strains. Int J Pharm Drug Anal. 2014;2:65-9.

11. Malinow M, McLaughlin P, Stafford C, Livingston AL, Kohler GO, Cheeke PR. Comparative effects of alfalfa saponins and alfalfa fiber on cholesterol absorption in rats. Am J Clin Nutr. 1979;32(9):1810-2. PMID: 474470 
12. Amraie E, Farsani MK, Sadeghi L, Khan TN, Babadi VY, Adavi Z. The effects of aqueous extract of alfalfa on blood glucose and lipids in alloxan-induced diabetic rats. Interv Med Appl Sci. 2015;7(3):124-8. doi: 10.1556/1646.7.2015.3.7

13. Elakovich SD, Hampton JM. Analysis of coumestrol, a phytoestrogen, in alfalfa tablets sold for human consumption. J Agric Food Chem. 1984;32(1):173-5. PMID: 6707329

14. Farsani MK, Amraie E, Kavian P, Keshvari M. Effects of aqueous extract of alfalfa on hyperglycemia and dyslipidemia in alloxan-induced diabetic Wistar rats. Interv Med Appl Sci. 2016;8(3):103-8. doi: 10.1556/1646.8.2016.3.5.

15. Hong Y-H, Chao W-W, Chen M-L, Lin B-F. Ethyl acetate extracts of alfalfa (Medicago sativa L.) sprouts inhibit lipopolysaccharide-induced inflammation in vitro and in vivo. J Biomed Sci. 2009;16(1):64. doi: 10.1186/1423-0127-16-64

16. Asgary S, Moshtaghian J, Hosseini M, Siadat H. Effects of alfalfa on lipoproteins and fatty streak formation in hypercholesterolemic rabbits. Pak J Pharm Sci. 2008;21(4) :460-4. PMID: 18930871

17. Mehranjani M, Shariatzadeh M, Desfulian A, Noori M, Abnosi M, Moghadam Z. Effects of Medicago sativa on nephropathy in diabetic rats. Indian $\mathbf{J}$ Pharm Sci. 2007;69(6):768-772.

DOI: 10.4103/0250-474X.39431

18. Marino M, Bersani C, Comi G. Antimicrobial activity of the essential oils of Thymus vulgaris L. measured using a bioimpedometric method. J Food Prot. 1999;62(9):1017-23. PMID: 10492476

19. Gradwohl RBH, Sonnenwirth AC, Jarett L. Gradwohl's clinical laboratory methods and diagnosis: Mosby; 1980.

20. Forbes B, Sahm D, Weissfeld A, Bailey Ss. Diagnostic microbiology 12th Edition: Mosby Elsevier, St. Louis, MO. 2007:778-81.

21. Chua LS. A review on plant-based rutin extraction methods and its pharmacological activities. J Ethnopharmacol. 2013;150(3):805-17.

doi: 10.1016/j.jep.2013.10.036.

22. Nostro A, Germano M, D'angelo V, Marino A, Cannatelli M. Extraction methods and bioautography for evaluation of medicinal plant antimicrobial activity. Lett Appl Microbiol. 2000;30(5):379-84. PMID: 10792667

23. Chavan SS, Jadhav RS, Khemnar KS, Tambe VB. Evaluation of antibacterial activity and phytochemical screening of Medicago sativa leaves. Int J Curr Res Acad Rev. 2015;3(5):308-13.

24. Sadowska B, Budzyńska A, Więckowska-Szakiel M, Paszkiewicz M, Stochmal A, Moniuszko-Szajwaj B, et al. New pharmacological properties of Medicago sativa and Saponaria officinalis saponin-rich fractions addressed to Candida albicans. J Med Microbiol. 2014;63(8):1076-86. doi: 10.1099/jmm.0.075291-0

25. Doss A, Parivuguna V, Vijayasanthi M, Surendran S. Antibacterial evaluation and phytochemical analysis of Medicago sativa L. against some microbial pathogens. Indian J Sci Technol. 2011;4(5):550-2.

26. Aliahmadi A, Roghanian R, Emtiazi G, Ghassempour A. A simple method for primary screening of antibacterial peptides in plant seeds. Iran J Microbiol. 2011;3(2):104-8.
PMID: 22347591

27. EL-Kamali HH, EL-Amir MY. Antibacterial activity and phytochemical screening of ethanolic extracts obtained from selected Sudanese medicinal plants. Current Research J Biological Sciences. 2010;2(2):143-6. 